\title{
Concrete fatigue experiment for sensor prototyping and validation of industrial SHM trials
}

\author{
Jack McAlorum, Grzegorz Fusiek, Tim Rubert, Paweł Niewczas \\ Electronic \& Electrical Engineering Department, University of Strathclyde, Glasgow, United Kingdom \\ g.fusiek@strath.ac.uk
}

\begin{abstract}
In this paper, preliminary results from a concrete fatigue experiment using a custom built machine are demonstrated. A pre-cracked concrete member is instrumented with bespoke metallic-bonded and epoxy-bonded fiber Bragg grating (FBG) displacement sensors, retrofitted over the crack. Fatigue loading is applied to the beam, with cycle magnitudes replicating results from a previous industrial trial concerning structural health monitoring (SHM) of a wind turbine foundation. Results are compared to an FEM model for verification. The new metallic-bonded crack displacement sensor design is compared in performance with the traditional epoxy-bonded design. Both sensors were sufficiently resilient under dynamic loading to successfully undergo $10^{5}$ cycle fatigue test. The sensors display a linear relationship with respect to one another; however, from the initial thermal characterization of the devices between 20 and $65^{\circ} \mathrm{C}$, the epoxy-bonded sensor exhibited considerable drift with every subsequent temperature cycle while the metallic-bonded construction was stable within the experimental error. The set up can be used over a long term to validate in situ results from distributed SHM sensors and for initial testing of sensors and data analytics strategies prior to any future field installations.
\end{abstract}

Keywords - concrete fatigue, machine, metallic-bonded, epoxybonded, FBG, fatigue test-bench

\section{INTRODUCTION}

Structural health monitoring (SHM) of civil infrastructure has evolved to include sensor systems to continually monitor key measurands [1]. Usually these are mechanical in nature, such as strain gauges applied in bridge cables [2], jet airframes [3] or critical members within a building [4]. Displacement sensors have been applied to cracks in concrete structures for SHM purposes [5]-[7] and used to determine deterioration [8].

Sensors used can vary from electrical- to optical-based, or even use wave propagation techniques [9]-[11]. Among opticalbased sensors, fiber Bragg gratings (FBGs) are arguably the most commonly used for SHM applications due to their flexibility and multiplexing ability [12]. FBGs can be implemented as strain, temperature or displacement sensors, commonly attached using epoxy, electroplating or entirely metallic methods [13]-[15].

Data from sensors in SHM campaigns are often required to be validated in closely simulated conditions. Furthermore, strategies for data analytics often need to be developed based on predictable and repeatable conditions, unlike those specific to a particular industrial trial. In some cases, a finite element method (FEM) or mathematical model is used for comparison to in situ measurements [16]-[17]. However, for a complex reinforced concrete structure, such as a wind turbine foundation, this approach may involve significant simplifications, rendering the final conclusions inaccurate. We therefore propose that an experimental test-bench can be used to replicate in situ conditions, verify sensor measurements and assist in experimentation involving data analytics. A combination of FEM and laboratory verification would provide more impactful evidence regarding any conclusions from the SHM analysis.

The motivation of this work follows the successful industrial application of crack displacement monitoring on an onshore wind turbine foundation [7]-[8]. To validate conclusions from this work, it was desired to carry out accelerated life experiments on a cracked specimen using similar loading. Since cracks are unpredictable, they are difficult to model both mathematically and using FEM. Producing similar characteristics in an experimental environment may provide a clearer understanding of the results. To do this, a highly customizable bi-directional (opening and closing of crack) load cycle is required.

In this paper, preliminary results from a concrete fatigue experiment using a custom built machine are demonstrated. A pre-cracked concrete member is instrumented with an in-house manufactured metallic-bonded and an epoxy-bonded FBG displacement sensors; retrofitted over the crack. Fatigue loading is applied to the beam, with magnitude based on results from a previous industrial application. Results are compared to an FEM model for verification. The two crack displacement sensor designs are compared in performance. Such an experimental set up can be used over a long term to validate in situ results. Furthermore, the test-bench can be used for initial testing prior to any future installations.

\section{SENSORS DESIGN AND FABRICATION}

Strain transducers in this work are FBG optical sensors. FBGs present many advantages over the electrical alternatives, such as: smaller size, multiplexing ability, immunity to electromagnetic interference and linear response to both strain and temperature [18].

\section{A. Fiber Bragg gratings}

FBG sensors comprise of a single core optical fiber with a section of modulating refractive index inscribed by UV light. Upon illumination by a broadband light source, this grating causes a short band wavelength reflection, the center of which is known as the Bragg wavelength, $\lambda_{B}$. Grating characteristics such as the period between index modulations are dependent on 
physical phenomenon acting upon the FBG; therefore, the Bragg wavelength is also affected. These variations $\left(\Delta \lambda_{B}\right)$ are dominated by strain and temperature, providing the following relationship:

$$
\frac{\Delta \lambda_{B}}{\lambda_{B}}=K_{\varepsilon} \varepsilon+K_{T} \Delta T
$$

where $K_{\epsilon}$ and $K_{T}$ are the FBG strain and temperature sensitivities respectively, $\epsilon$ is the applied strain and $\Delta T$ is the change in temperature.

To measure mechanical strain with an FBG, the sensor requires temperature compensation which is often realized by using an additional FBG temperature sensor decoupled from mechanical strain or by the sensor thermal characterization.

\section{B. Crack displacement sensors}

A displacement sensor is formed by utilizing an FBG-based strain sensor firstly bonded to a substrate, which is then attached to the specimen between two anchor points of gauge length $L_{g}$ apart. The displacement between the anchor points, $\Delta L$, can then be extracted as:

$$
\frac{\Delta L}{L_{g}}=\frac{\Delta \lambda_{B}}{K_{\varepsilon} \lambda_{B}}-K_{T} \Delta T
$$

Two variations of displacement sensor design are considered in this work: a metallic-bonded construction of FBG to substrate and a construction involving an epoxy-bonded FBG to a similar substrate. Epoxy-based attachment is popular for strain and displacement sensors due to the ease of installation and relatively reliable performance. We have previously shown evidence of humidity dependence for some epoxies [19], which may make them unsuitable for some applications. Metallicbonded sensors were shown to be immune to such effects.

To fabricate the metallic-bonded FBG displacement sensor, shown in Fig. 1, a standard 10-mm long FBG inscribed in a 15$\mathrm{mm}$ bare section of a single-mode copper coated fiber was prestressed and encapsulated in a kovar capillary (length of $35 \mathrm{~mm}$, OD $0.7 \mathrm{~mm}$ ). The kovar capillary, copper-coated fiber and magnetic stainless steel shims are then brazed together at around $620^{\circ} \mathrm{C}$ using a silver-based brazing paste and an induction heating system [15]. The assembly was then cooled down naturally to room temperature. Following the FBG encapsulation process, the sensor was attached to a stainless steel plate using spot welding. The plate was equipped with M4 bolts that were brazed to the plate before spot welding the strain sensor. The distance between the anchoring bolts was $100 \mathrm{~mm}$.

The epoxy-bonded displacement sensor, shown in Fig. 2, shared a similar construction as the metallic sensor. The utilized FBG was not encapsulated in a kovar capillary but bonded directly to a stainless steel plate with M-Bond AE-10 epoxy adhesive. The plate was equipped with M4 anchoring bolts spaced $100 \mathrm{~mm}$ apart.

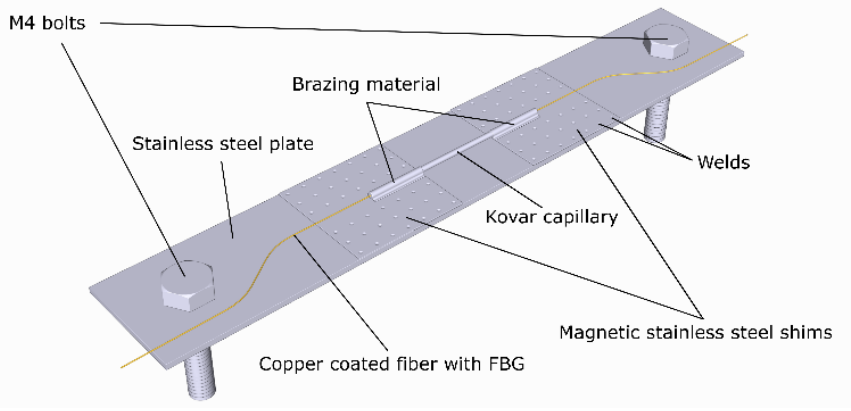

Fig. 1. Metallic-bonded displacement sensor.

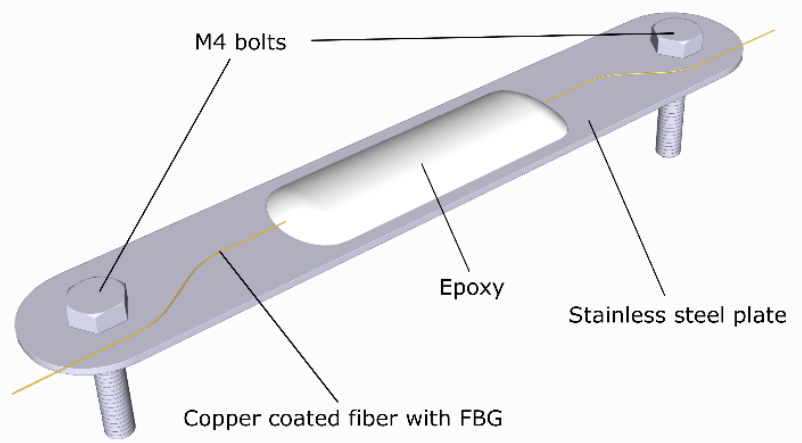

Fig. 2. Epoxy-bonded displacement sensor.

\section{THERMAL CHARACTERISATION AND CALIBRATION}

To determine thermal sensitivities of the displacement sensors and to allow for their temperature compensation, the devices were thermally characterized before their attachment to the concrete structure [12].

The response of the epoxy-bonded and metallic-bonded displacement sensors when thermally cycled between $20^{\circ} \mathrm{C}$ and $65^{\circ} \mathrm{C}$ are shown in Fig. 3 and Fig. 4 respectively.

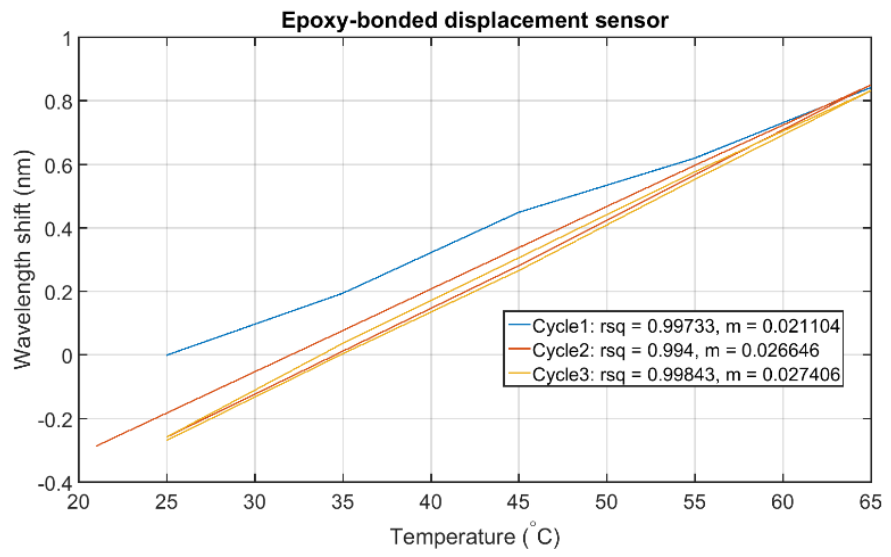

Fig. 3. Temperature response of a epoxy-bonded strain sensor thermally cycled three times. Legend shows linear fit, $R^{2}$ and gradient, $m$ values for each cycle. 


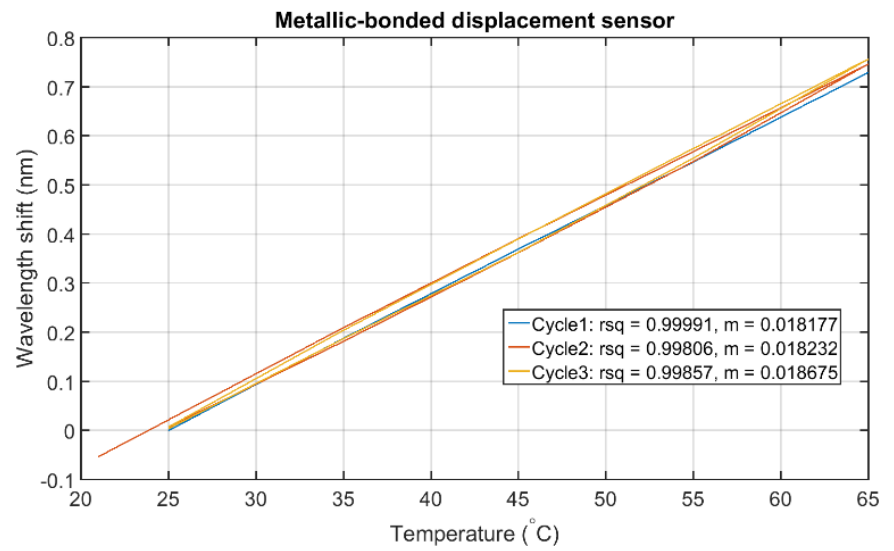

Fig. 4. Temperature response of a metallic-bonded sensor thermally cycled three times. Legends show linear fit, $R^{2}$ and gradient, $m$ values for each cycle.

The epoxy-bonded displacement sensor has slightly higher sensitivity to temperature than the metallic equivalent (greater gradient, $m$ ). The metallic-bonded sensor has better linearity and repeatability after three cycles while the epoxy-bonded sensor response drifts significantly with every subsequent temperature cycle. Clearly, the epoxy-bonded sensor requires thermal cycling to stabilize its response before it can be reliably used.

The thermal sensitivity coefficients obtained from the characterization described above were used for temperature compensation of the displacement sensors.

\section{FATIGUE IN CONCRETE}

Fatigue refers to damage that occurs in a specimen under repetitive cyclic loading at a magnitude lower than the ultimate strength [22]. In this work, the displacement sensors are attached to a concrete beam specimen which is placed in a bespoke fatigue testing machine, previously developed by the authors [21].

The concrete beam specimen considered here has a cross section of $100 \times 100 \mathrm{~mm}$ and a length, $L$, of $950 \mathrm{~mm}$ to provide room for sensors cabling. The beam is reinforced with two 6 $\mathrm{mm}$ diameter steel rebar placed $25 \mathrm{~mm}$ from the bottom and top surfaces in the center of the beam. During curing, a deliberate notch of width $1 \mathrm{~mm}$ and $5 \mathrm{~mm}$ deep is created on the top surface to imitate a crack.

\section{A. Fatigue testing machine}

An extensive presentation of the design and specification for the fatigue machine has previously been published [21], where the machine capability for testing fatigue of a metal beam was presented. The machine has not been used for concrete experimentation until this point.

The machine is capable of providing bi-directional 4-point bending loads to a $1 \mathrm{~m}$ long beam specimen with a maximum force of $\pm 25 \mathrm{kN}$. The machine itself consists of a large steel frame to house the actuator, with a smaller frame providing the 4-point attachment between actuator and beam. 4-point bending provides room for a deliberate crack to be introduced and for sensors to be placed on top. Fig. 5 shows the overall set up. The frames were previously simulated using FEM modelling to ensure a long-term/infinite life was possible [21].

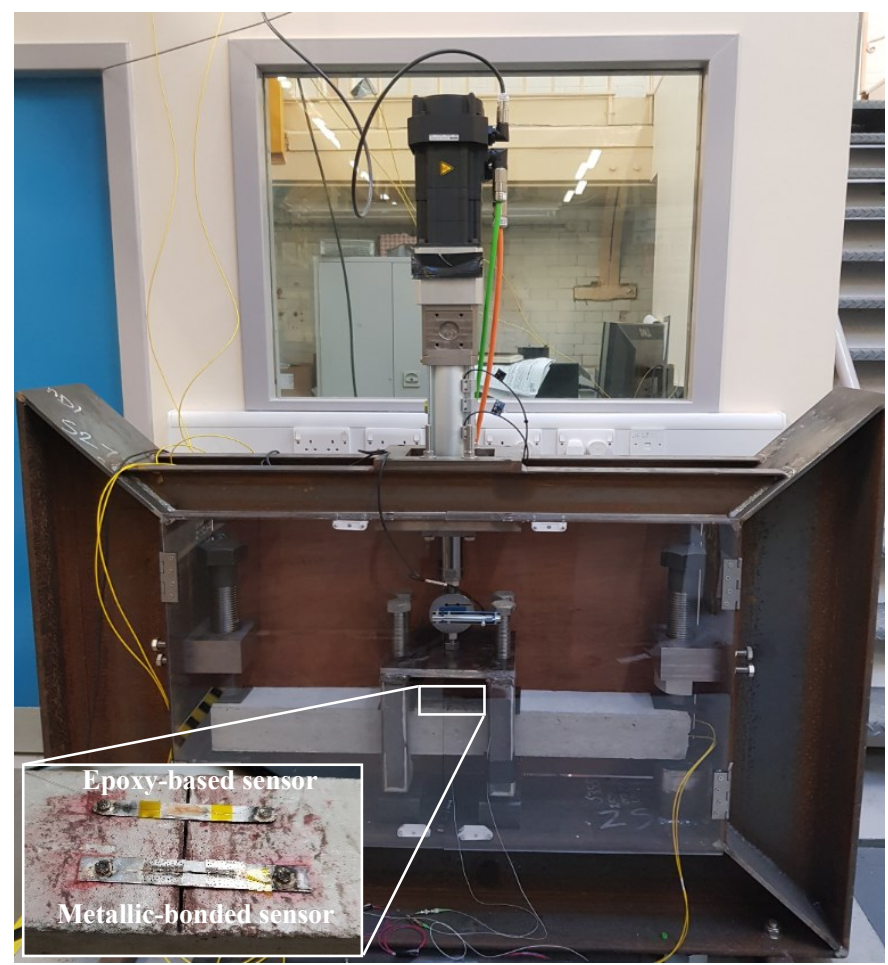

Fig. 5. Machine design and experimental set up. Insert: sensor instrumentation over notch.

\section{B. Concrete beam mathematical approach}

As mentioned earlier, the aim of this work is to develop a methodology for validating conclusions from the industrial trial involving SHM of a wind turbine foundation. This will be achieved by carrying out accelerated life experiments on a cracked specimen using similar loading. To estimate the required force to produce crack displacements in the concrete beam similar to the industrial application, a simplified 2D mathematical approach is considered and the results are verified by $3 \mathrm{D}$ FEM modelling of the setup

The following simplifications and assumptions in this mathematical approach are made. Firstly, to reduce calculation complexity, it is assumed that concrete is not reinforced. Secondly, load is applied as point loads to, again, simplify calculations. In the real world, the load is applied using a steel frame. This will cause frictional losses and the final applied load will be reduced. During the industrial application, cracks displaced between $\pm 3 \mu \mathrm{m}$. To acquire similar displacements during the experiment, we can initially calculate the required force. Firstly, converting to strain using the intended sensor gauge length $L_{g}=100 \mathrm{~mm}$, gives the required strain on the center face of the beam $\epsilon_{f}=30 \mu \epsilon$ and therefore required stress $\sigma_{f}=0.51 \mathrm{MPa}$. Since 4-point bending is used, we can model the experiment as a fixed beam with two point loads, as shown in Fig. 6. 


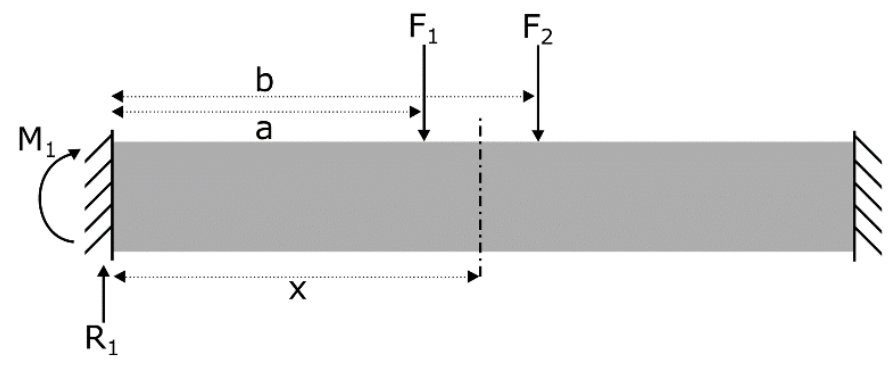

Fig. 6. 4-point bending diagram.

Here, $F_{1}=F_{2}=0.5 F_{a}$ where $F_{a}$ is the required force applied by the actuator, $a=375 \mathrm{~mm}, b=575 \mathrm{~mm}$ and $x=475 \mathrm{~mm}$ is the location of $\sigma_{f}$. Since the forces are equal and the $x$ position is centered between forces, the stress by each point load will be equal. In other words, $\sigma_{f}=2 \sigma_{F 1}$. Therefore, stress caused by $F_{l}$, $\sigma_{F 1}=0.255 \mathrm{MPa}$. From this we can calculate the required moment, $M_{x F l}$, at point $x$ by force $F_{l}$ as [20]:

$M_{x F 1}=\frac{\sigma_{F 1} 2 I_{s}}{H}$

where $I_{\mathrm{s}}=8.33 \times 10^{-6} \mathrm{~m}^{3}$ is the area moment of inertia for a square cross section beam with height $H=0.1 \mathrm{~m}$. This means required moment at point $x$ due to force $F_{l}$ is $42.483 \mathrm{Nm}$. The moment can then be related to force using the equations for the fixed beam:

$$
M_{x F 1}=M_{1}+R_{1} x-F_{1}(x-a)
$$

where $M_{l}$ is the reaction moment and $R_{l}$ is the reaction force. This can be expanded to provide the force, $F_{l}$ in relation to moment $M_{x F 1}$ as:

$$
F_{1}=\frac{M_{x F 1}}{\frac{-a(L-a)^{2}}{L^{2}}+\frac{(L-a)^{2}(L+2 a) x}{L^{3}}-(x-a)}
$$

Substituting provides a required force, $F_{l}=574 \mathrm{~N}$ and thus an actuator force, $F_{a}=1148 \mathrm{~N}$.

\section{FEM model}

An FEM model was used to simulate the 4-point frame and beam interaction, applying the relevant force to the beam. In the mathematical approach, the force is applied using a point load rather than the frame dimensions. The approach also does not take into account the influence of rebars on the structure. Applying this force in an FEM environment will produce values closer to the real world. The FEM simulation geometry is shown in Fig. 7 and the strain distribution during a force of $1148 \mathrm{~N}$ applied to the frame is shown in Fig. 8. Steel reinforcement in the beam was represented by the truss model.

Results match the calculations with a small loss of $0.24 \mu \epsilon$, providing confidence in performing an experiment using the calculated force to achieve the required crack displacement.

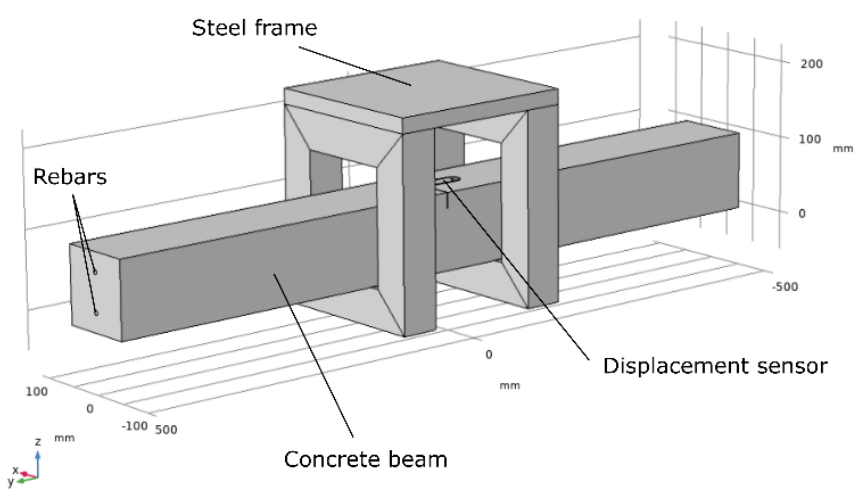

Fig. 7. FEM simulation geometery of 4-point bending frame and beam with rebars.

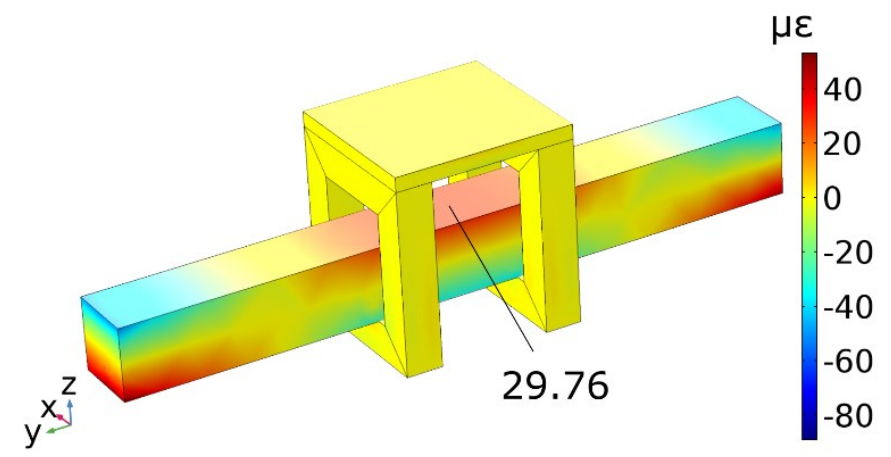

Fig. 8. FEM simulation showing tension strain in y-axis during $1148 \mathrm{~N}$ force applied to top of 4-point frame in positive $\mathrm{z}$ direction.

\section{FATIGUE EXPERIMENT AND RESULTS}

To validate the theoretical considerations discussed above and to assess the ability of the set-up to perform long-term fatigue experiments, the concrete beam with a $1 \mathrm{~mm}$ notch was instrumented with epoxy-bonded and metallic-bonded FBG displacement sensors and placed in the fatigue machine as described earlier. A cyclic load of $\pm 1148 \mathrm{~N}$ was applied to the frame by means of an actuator at approximately $1 \mathrm{~Hz}$ and the sensors response was monitored optically using a commercial interrogator at $2 \mathrm{kHz}$. A short snapshot of data from the displacement sensors during ongoing fatigue cycling are shown in Fig. 9. In the whole experiment, a total of $10^{5}$ cycles were run and both sensors showed no apparent degradation.

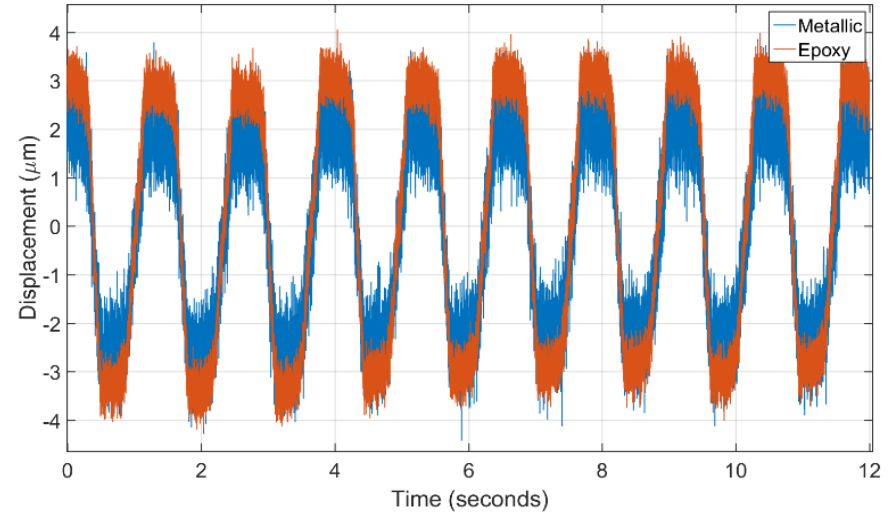

Fig. 9. Displacement detected by metallic and epoxy sensors during load cycles. 
From the above results, it can be seen that the experimental set up matches both the calculations and the FEM simulations, with the applied force providing required crack displacements of $\pm 3 \mu \mathrm{m}$. When comparing the sensors performance, it can be observed that the metallic sensor has a slightly smaller strain sensitivity in comparison to the epoxy-based sensor. However, when the responses from both sensors are plotted in a single graph, a linear relationship is apparent, as shown in Fig. 10. The symmetry in response represents a relatively constant error in measurements, which is due to equipment resolutions. This linear relationship confirms the performance of the metallicbonded sensor. Considering the additional benefits over epoxybonded sensor, such as temperature response reliability and immunity to humidity [19], a metallic-bonded approach may be preferred for some applications.

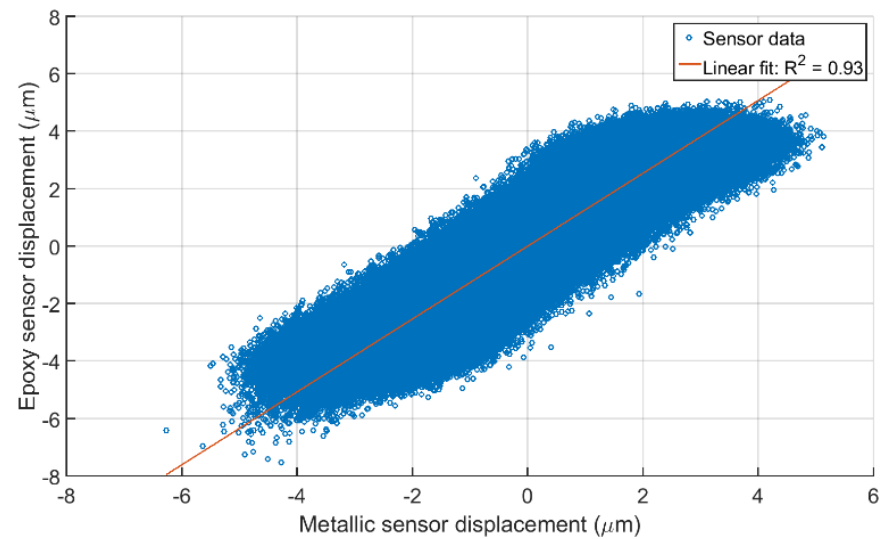

Fig. 10. Linear relationship between the responses of metallic and epoxy-based sensors.

It should be noted that due to the used actuator controller, the force resolution is $144.6 \mathrm{~N}$ which is equivalent to crack displacement resolution of $0.8 \mu \mathrm{m}$. This does not take into account noise of the optical system and individual sensors sensitivities.

\section{CONCLUSIONS}

This paper has presented preliminary results for metallicbonded and epoxy-bonded optical displacement sensors designed specifically for structural health monitoring in civil engineering applications. The sensors and the proposed experimental set-up could be used to monitor fatigue in concrete structures and to validate industrial conclusions by closely replicating field conditions. It was shown via laboratory experiments that the bespoke metallic-bonded FBG sensor performs linearly with reference to an epoxy-bonded sensor despite a slightly smaller strain and temperature sensitivities. However, from the thermal characterization of the devices, the metallic-bonded sensor showed a reliable, zero-drift performance over the epoxy-bonded alternative. Furthermore, both sensors were sufficiently resilient under dynamic loading to successfully undergo $10^{5}$ cycles of fully reversed $\pm 30 \mu \mathrm{\epsilon}$ fatigue test on a concrete specimen without apparent deterioration in performance. It was also shown that the designed fatigue test-bench when combined with the considered sensors has the potential to form a useful tool for validating in-situ measurements and for initial testing of newly developed sensors prior to field installations. For future work, the set up will be used to apply more complex loading cycles, closely replicating wind turbine start/stop procedures and other loading scenarios to validate the results from the field trial.

\section{ACKNOWLEDGEMENTS}

Research presented in this paper was funded by the industrial partners SSE and SPR, and the EPSRC, grant number EP/L016680/1.

We gratefully acknowledge the help of Dr Marcus Perry of Civil and Environmental Engineering Department, University of Strathclyde, with providing access to the concrete beam manufacturing facilities in the departmental laboratory.

\section{REFERENCES}

[1] M. Abdo, Structural health monitoring, history, applications and future. A review book. Open Science, 2014.

[2] J. M. Ko and Y. Q. Ni, "Structural health monitoring and intelligent vibration control of cable-supported bridges: Research and application," KSCE Journal of Civil Engineering 7(6):701-716, 2003.

[3] S. Hunt and I. Hebden, "Validation of the eurofighter typhoon structural health and usage monitoring system," Smart materials and structures, September 2001.

[4] Y. Ni, Y. Xia, and X. Ye, "Structural health monitoring of a tall building with huge floating platform," Advances in Science and Technology, September 2012.

[5] H. Lima et al., "Structural health monitoring of the church of Santa Casa da MisericOrdia of Aveiro using FBG sensors," IEEE Sensors Journal, vol. 8, no. 7, pp. 1236-1242, 2008.

[6] N. A. Hoult, P. R. A. Fidler, P. G. Hill, and C. R. Middleton, "Long-term wireless structural health monitoring of the ferriby road bridge," Journal of Bridge Engineering, 2010.

[7] M. Perry, J. McAlorum, G. Fusiek, P. Niewczas and T. Rubert, "Crack monitoring of operational wind turbine foundations", MDPI Sensors, August 2017.

[8] J. McAlorum, M. Perry, G. Fusiek, P. Niewczas, I. McKeeman and T. Rubert, "Deterioration of cracks in onshore wind turbine foundations", Engineering Structures, 167, May 2018.

[9] J. Wilson and T. Kenny, Sensor Technology Handbook. Elsevier, 2005.

[10] B. Glisic and D. Inaudi, Fibre Optic Methods for Structural Health Monitoring. John Wiley \& Sons, 2007.

[11] T. Stepinski, T. Uhl, and W. Staszewski, Advanced structural damage detection: from theory to engineering application. John Wiley \& sons, 2013.

[12] Y. Rao, "In-fibre Bragg grating sensors," Measurement Science and Technology, vol. 8, no. 4, pp. 355-375, 1997.

[13] Li, E., Xi, J., Chicharo, J.F., Liu, T., Li, X., Jiang, J., Li, L., Wang, Y., Zhang, Y., "The Experimental evaluation of FBG sensor for strain measurement of prestressed steel strand," Proc. SPIE Smart Structures Devices and Systems II 5649, 463-469 (2004)

[14] Yun Tu and Shan-Tung Tu, "Fabrication and characterization of a metalpackaged regenerated fiber Bragg grating strain sensor for structural integrity monitoring of high-temperature components", Smart Mater. Struct. 23(3) (2013)

[15] P. Niewczas and G. Fusiek, "Induction heating assisted optical fiber bonding and sealing technique," in 21 st International Conference on Optical Fiber Sensors, 2011, p. 77536H.

[16] J. M. W. Brownjohn, M. Bocciolone, A. Curami, M. Falco, and A. Zasso, "Humber bridge full-scale measurement campaigns 1990-1991," Journal of Wind Engineering and Industrial Aerodynamics, vol. 52, no. C, pp. 185-218, 1994 
[17] C. P. Fritzen and K. Bohle, "Application of model-based damage identification to a seismically loaded structure," Smart materials and structures, 2001.

[18] K. O. Hill and G. Meltz, "Fiber bragg grating technology fundamentals and overview," Journal of lightwave technology, vol. 15, no. 8, 1997.

[19] J. McAlorum, T. Rubert, G. Fusiek, I. McKeeman, L. Clayburn, M. Perry and P. Niewczas, "Comparison of epoxy and braze-welded attachment methods for FBG strain," in 16th IEEE International Conference on Sensors, Glasgow, 2017.
[20] W. C. Young and R. G. Budynas, Roark's Formulas for Stress and Strain, 7 ed., McGraw-Hill, 2002, pp. 125 - 267.

[21] J. McAlorum, T. Rubert, G. Fusiek, P. Niewczas and G. Zorzi, "Design and demonstration of a low-cost small-scale fatigue testing machine for multi-purpose testing of materials, sensors and structures," MDPI Machines, vol. 6, 2018.

[22] Y. Lee, J. Pan, R. Hathaway and M. Barkey, "Fatigue Damage Theories," Fatigue Testing and Analysis, 2005. 